\title{
Two-band superconductivity with unconventional pairing symmetry in $\mathrm{HfV}_{2} \mathrm{Ga}_{4}$
}

\author{
A. Bhattacharyya $\odot,{ }^{1, *}$ P. P. Ferreira $\odot,{ }^{2, \dagger}$ F. B. Santos, ${ }^{3}$ D. T. Adroja $\odot,{ }^{4,5}, \$$ J. S. Lord $\odot,{ }^{4}$ L. E. Correa, ${ }^{3}$ A. J. S. Machado, ${ }^{3}$ \\ A. L. R. Manesco (i),,$^{2,6}$ and L T. F. Eleno $\mathbb{1}^{2, \S}$ \\ ${ }^{1}$ Department of Physics, Ramakrishna Mission Vivekananda Educational and Research Institute, Belur Math, \\ Howrah 711202, West Bengal, India \\ ${ }^{2}$ Computational Materials Science Group (ComputEEL), Materials Engineering Department (Demar), Escola de Engenharia de Lorena, \\ Universidade de São Paulo (EEL-USP), Lorena-SP, Brazil \\ ${ }^{3}$ Materials Engineering Department (Demar), Escola de Engenharia de Lorena, Universidade de São Paulo (EEL-USP), Lorena-SP, Brazil \\ ${ }^{4}$ ISIS Facility, Rutherford Appleton Laboratory, Chilton, Didcot, Oxon OX11 0QX, United Kingdom \\ ${ }^{5}$ Highly Correlated Matter Research Group, Physics Department, University of Johannesburg, Auckland Park 2006, South Africa \\ ${ }^{6}$ Kavli Institute of Nanoscience, Delft University of Technology, Delft, The Netherlands
}

(Received 24 September 2019; accepted 26 February 2020; published 1 April 2020)

\begin{abstract}
In this Rapid Communication, we have examined the superconducting ground state of the $\mathrm{HfV}_{2} \mathrm{Ga}_{4}$ compound using resistivity, magnetization, zero-field (ZF), and transverse-field (TF) muon-spin relaxation and rotation ( $\mu \mathrm{SR}$ ) measurements. Resistivity and magnetization unveil the onset of bulk superconductivity with $T_{\mathrm{C}} \sim 3.9 \mathrm{~K}$. TF- $\mu$ SR measurements show the temperature dependence of the superfluid density, indicating, surprisingly, a nodal two-gap $(s+d)$-wave superconducting order parameter. In addition, the ZF muon relaxation rate increases with decreasing temperature below $4.6 \mathrm{~K}$, suggesting the presence of weak spin fluctuations. These observations pointed to an unconventional multiband nature of the superconducting ground state. To better understand these findings, we carry out first-principles electronic-structure calculations, further highlighting multiple disconnected sheets with very different orbital weights and spin-orbit coupling composing the Fermi surface, bridging the way for a nodal multiband superconductivity scenario. In this vein, therefore, the $\mathrm{HfV}_{2} \mathrm{Ga}_{4}$ family stands out as an open avenue to novel unexplored unconventional superconducting compounds and an ideal playground to investigate the mechanisms behind such phenomena.
\end{abstract}

DOI: 10.1103/PhysRevResearch.2.022001

Unconventional superconducting materials have attracted considerable interest over the last decade due to their extremely rich physics and emerging breakthrough properties $[1,2]$. In such materials, the superconducting state transcends the BCS-like signatures, as well as the isotropic $s$-wave pairing symmetry of the gap structure. Instead, a complex interaction framework among electrons, the crystal lattice, and spin-orbital fluctuations are established as the possible mediation mechanism of the Cooper pairs [3]. $\mathrm{HfV}_{2} \mathrm{Ga}_{4}$ is a newly discovered superconducting compound with a critical temperature $\left(T_{\mathrm{C}}\right)$ of $\approx 3.9 \mathrm{~K}$ that crystallizes in the tetragonal bodycentered prototype $\mathrm{YbMo}_{2} \mathrm{Ga}_{4}$ [4]. Substantial deviations of the temperature dependence of upper and lower critical fields from the expected Werthamer-Helfand-Hohenberg formula [5] has led the authors to argue in favor of the presence of two superconducting gaps in the Fermi surface [4]. Later,

\footnotetext{
*amitava.bhattacharyya@rkmvu.ac.in

†pedroferreira@usp.br

¥devashibhai.adroja@stfc.ac.uk

§luizeleno@usp.br
}

Published by the American Physical Society under the terms of the Creative Commons Attribution 4.0 International license. Further distribution of this work must maintain attribution to the author(s) and the published article's title, journal citation, and DOI. first-principles electronic-structure calculations revealed the presence of electrons occupying very distinct bands at the Fermi level in the presence of spin-orbit coupling (SOC) effects [6] and a substantial elastic anisotropy regime due to the presence of extended linear vanadium chains in the structure [7]. These features, in a first analysis, indicate the feasibility of multiband superconductivity and pronounced anisotropic properties. Additionally, V-based superconductors $[8,9]$ are usually mediated by an electron-phonon coupling mechanism, showing a conventional BCS behavior without expressive spin-fluctuation manifestations.

In this Rapid Communication, we present unambiguous evidence of two-gap superconductivity in $\mathrm{HfV}_{2} \mathrm{Ga}_{4}$ using transverse-field (TF) muon-spin rotation ( $\mu \mathrm{SR})$ measurement. However, in opposition to what was expected for V-based superconductors and from previous experimental and theoretical attempts, we have discovered an unconventional, and uncommon, superconducting order parameter with $(s+d)$-wave pairing symmetry and experimental indications of spin fluctuations. These experimental findings are further supported by density functional theory (DFT) calculations. Therefore, $\mathrm{HfV}_{2} \mathrm{Ga}_{4}$ could represent a novel family of unconventional superconductors, going beyond Fe-based compounds, heavy fermions, noncentrosymmetric systems, and other known classes [1-3]. Thus, several compounds within the $\mathrm{HfV}_{2} \mathrm{Ga}_{4}$ family, such as $\mathrm{ScV}_{2} \mathrm{Ga}_{4}, \mathrm{ZrV}_{2} \mathrm{Ga}_{4}$ [6,7], and many rareearth-based materials, stand out as an open avenue and an 

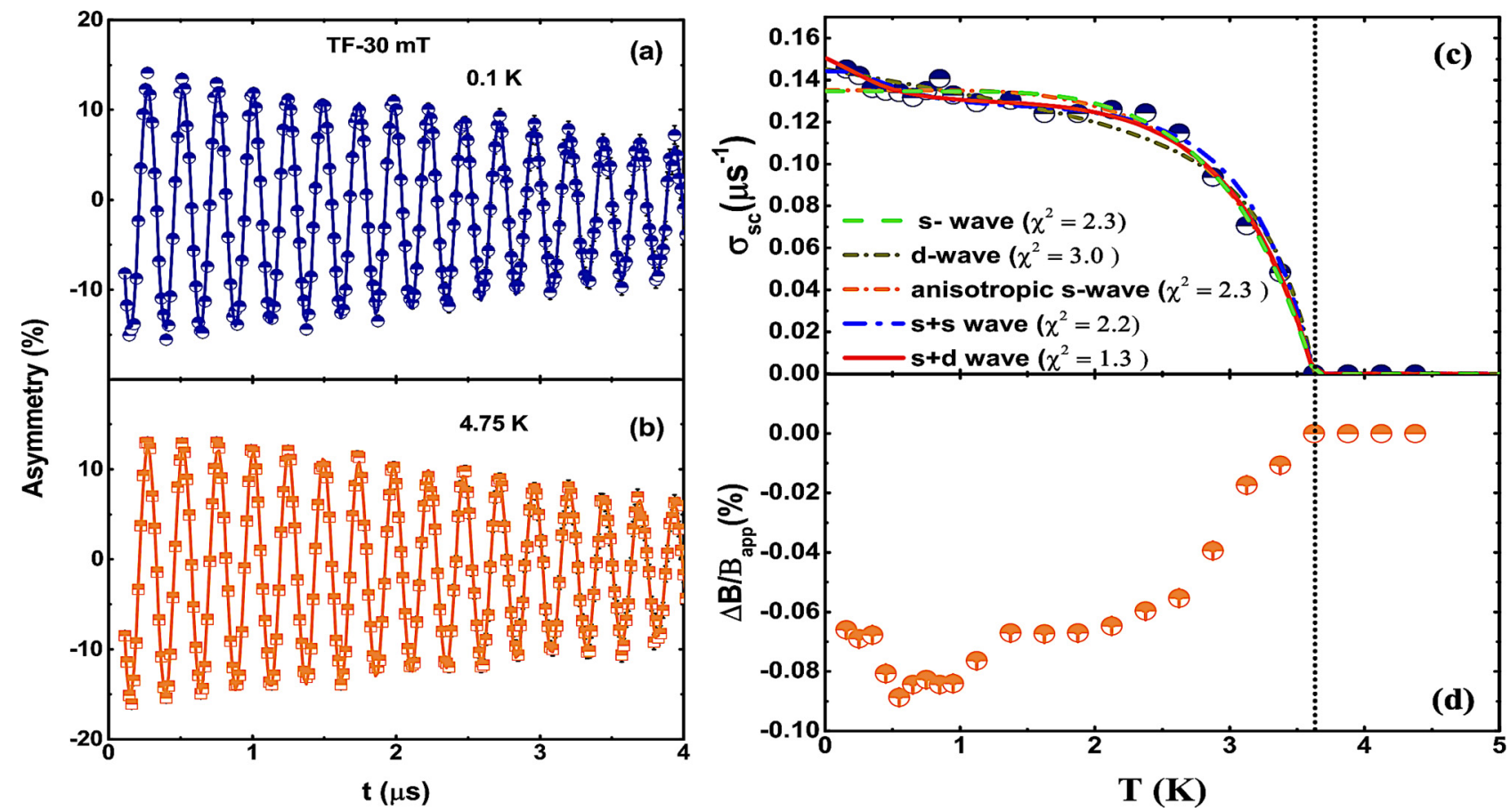

FIG. 1. Representative TF- $\mu$ SR asymmetry spectra in the low time region collected at (a) $T=0.1 \mathrm{~K}$ and (b) $T=4.75 \mathrm{~K}$ (i.e., below and above $T_{\mathrm{C}}$ ) in an applied magnetic field of $30 \mathrm{mT}$. (c) represents the $\sigma_{s c}(T)$ data in field-cooling mode with fits using various gap models. The dashed green line shows the fit using an isotropic single-gap $s$-wave model, and the solid red line and dash-dotted blue line show the fit to a two-gap model, $s+d$ wave, and $s+s$ wave, respectively. The orange dash-dotted line shows the fit using an anisotropic $s$-wave model and the dash-dotted purple line shows the fit using the $d$-wave model. (d) Temperature dependence of the normalized internal field from the sample.

ideal playground to investigate the nature and driven mechanisms of an unconventional superconducting ground state, since it is expected to find both conventional and unconventional superconductivity coexisting in the same material's class.

For the present $\mu$ SR study a high-quality polycrystalline sample with general stoichiometry $\mathrm{HfV}_{2} \mathrm{Ga}_{4}$ was prepared by arc melting on a water-cooled $\mathrm{Cu}$ crucible in a high-purity Ar atmosphere. The arc-melted pellet was encapsulated in an evacuated quartz ampoule and heated up to $800^{\circ} \mathrm{C}$, kept at that temperature for $1 \mathrm{wk}$, then quenched in cold water. In order to get more details about the crystal growth procedures and microstructure information, see Ref. [4]. Magnetotransport measurements were performed using a VSM-PPMS EverCool II from Quantum Design. A powdered sample of $\mathrm{HfV}_{2} \mathrm{Ga}_{4}$ was used for the $\mu \mathrm{SR}$ experiments, which were carried out on the MUSR spectrometer at the ISIS Pulsed Neutron and Muon Source of Rutherford Appleton Laboratory, UK. The sample was placed on a silver holder (99.999\%) using GE varnish, which was loaded in a dilution refrigerator operating between 0.1 and $4.75 \mathrm{~K}$. Zero-field (ZF) and TF- $\mu$ SR measurements were performed at different temperatures between 0.1 and $4.75 \mathrm{~K}$. For ZF- $\mu$ SR measurement, an active compensation system was used to cancel any stray magnetic fields at the sample space to a level of $\sim 10^{-4} \mathrm{mT}$. ZF- $\mu$ SR measurement is beneficial to identify the spontaneous internal field associated with time-reversal symmetry breaking [10]. TF- $\mu$ SR measurements were carried out in the presence of an external magnetic field of $30 \mathrm{mT}$, which is well above the lower critical field $\left(\mu_{0} H_{c 1}=1.2 \mathrm{mT}\right)$, and well below the upper critical field $\left(\mu_{0} H_{c 2}=1.1 \mathrm{~T}\right)$ of $\mathrm{HfV}_{2} \mathrm{Ga}_{4}$. The experimental data were analyzed using the WiMDA software [11].

Santos et al. [4] recently reported superconductivity on $\mathrm{HfV}_{2} \mathrm{Ga}_{4}$ at $T_{\mathrm{C}}=3.9 \mathrm{~K}$, in which the upper and lower critical fields show unusual dependence with reduced temperature $\left(T / T_{\mathrm{C}}\right)$, suggesting strong deviations from the conventional BCS behavior. This type of unusual behavior is also seen for other materials, such as $\mathrm{Cu}_{0.3} \mathrm{ZrTe}_{2-y}$ [12], fluorine-doped $\mathrm{NdFeAsO}$ [13], FeSe [14], $\mathrm{SmFeAsO}_{0.9} \mathrm{~F}_{0.1}, \mathrm{Ba}_{0.6} \mathrm{~K}_{0.4} \mathrm{Fe}_{2} \mathrm{As}_{2}$ [15,16], and $\mathrm{MgB}_{2}$ [17]. To clarify the microscopic characteristics and superconducting gap structure of $\mathrm{HfV}_{2} \mathrm{Ga}_{4}$, TF$\mu$ SR measurements were carried out in the mixed superconducting state. First, we applied an external magnetic field of $30 \mathrm{mT}$ above $T_{\mathrm{C}}$, followed by cooling down to $0.1 \mathrm{~K}$. The TF$\mu \mathrm{SR}$ asymmetry spectra were collected at various temperatures up to $T_{\mathrm{C}}$. Figures 1(a) and 1(b) exhibit two representative TF- $\mu$ SR spectra collected at $T=4.75$ and $0.1 \mathrm{~K}$ in $H=$ $30 \mathrm{mT}$. The asymmetry spectra at $0.1 \mathrm{~K}$ show faster relaxation compared to $4.75 \mathrm{~K}$ data as a result of the development of the flux line lattice below $T_{\mathrm{C}}$. The time evolution of the $\mu \mathrm{SR}$ asymmetry can be modeled by $A_{\mathrm{TF}}(t)=A_{1} \cos \left(\gamma_{\mu} B_{1} t+\right.$ $\Phi) \exp \left(-\frac{\sigma^{2} t^{2}}{2}\right)+A_{\mathrm{bg}} \cos \left(\gamma_{v} B_{\mathrm{bg}} t+\Phi\right)$ [18-21], where $A_{1}$ and $A_{\text {bg }}$ describe the initial asymmetries belonging to the sample and silver holder contributions, individually, with $A_{\mathrm{bg}}$ not undergoing any depolarization; $B_{1}$ and $B_{\mathrm{bg}}$ are the internal fields from the sample and from the sample holder, respectively. $\gamma_{\nu} / 2 \pi=135.53 \mathrm{MHz} / \mathrm{T}$ is the muon gyromagnetic ratio, $\Phi$ is the initial phase, and $\sigma$ is a Gaussian muon-spin relaxation rate. The flux line lattice related muon relaxation can be extracted by subtracting the nuclear contribution according 
TABLE I. The parameters obtained from the fit to the $\sigma_{\mathrm{sc}}(T)$ data of $\mathrm{HfV}_{2} \mathrm{Ga}_{4}$ using different gap models.

\begin{tabular}{|c|c|c|c|c|c|}
\hline Model & $g(\phi)$ & Gap value $\Delta(0)(\mathrm{meV})$ & Gap ratio $2 \Delta(0) / k_{\mathrm{B}} T_{\mathrm{C}}$ & Weight factor ${ }^{\mathrm{a}}$ & $\chi^{2}$ \\
\hline$s+d$ wave & $1, \cos (2 \phi)$ & $0.98(1) ; 0.09(6)$ & $6.27(2) ; 1.14(1)$ & $0.57(1)$ & $1.3(2)$ \\
\hline$s+s$ wave & 1 & $1.08(3) ; 0.09(7)$ & $6.91(1) ; 0.57(5)$ & $0.87(2)$ & $2.2(3)$ \\
\hline$s$ wave (anisotropy) & $\frac{|1+\cos (\phi)|}{2}$ & $1.14(2)$ & $7.29(2)$ & 1.0 & $2.3(1)$ \\
\hline$s$ wave & 1 & $0.91(4)$ & $5.84(1)$ & 1.0 & $2.3(2)$ \\
\hline$d$ wave & $\cos (2 \phi)$ & $9.78(7)$ & $2.82(1)$ & 1.0 & $3.0(3)$ \\
\hline
\end{tabular}

${ }^{a}$ The contribution from the first component of the gap function.

to $\sigma_{\mathrm{sc}}=\sqrt{\sigma^{2}-\sigma_{\mathrm{n}}^{2}}$, where $\sigma_{\mathrm{n}}$ is the nuclear magnetic dipolar contribution which is temperature independent and was obtained from spectra measured above $T_{\mathrm{C}}$. By fitting the spectra at $4.75 \mathrm{~K}$ we obtained $\sigma_{\mathrm{n}}=0.35 \mu \mathrm{s}^{-1}$. Considering that $\sigma_{\mathrm{sc}}$ is linked to the magnetic penetration $\operatorname{depth}(\lambda)$ by $\sigma_{\mathrm{sc}} \approx$ $1 / \lambda^{2}$, the superconducting gap symmetry can be determined from the temperature dependence of $\sigma_{\mathrm{sc}}(T)$. The temperature dependence of magnetic penetration depth was analyzed employing different models, generally described by $\frac{\sigma_{\mathrm{sc}}(T)}{\sigma_{\mathrm{sc}}(0)}=$ $\frac{\lambda^{-2}(T)}{\lambda^{-2}(0)}=1+\frac{1}{\pi} \int_{0}^{2 \pi} \int_{\Delta(T, \phi)}^{\infty}\left(\frac{\partial f}{\partial E}\right) \frac{E d E d \phi}{\sqrt{E^{2}-\Delta(T, \phi)^{2}}}[22,23]$, where $\Delta$ is an angle-dependent gap function, $f=\left[1+\exp \left(E / k_{B} T\right)\right]^{-1}$ is the Fermi function, and the integration signifies an average across the Fermi surface. The gap is expected to follow the function $\Delta(T)=\Delta_{0} \delta\left(T / T_{c}\right) g(\phi)$, where $\Delta_{0}$ is the maximum gap value at zero temperature and $g(\phi)$ is the angular dependence of the gap, equal to 1 and $\cos (2 \phi)$ for an $s$ - and $d$-wave model, respectively. Here $\phi$ is the azimuthal angle. The superconducting gap symmetry is expected to follow $\delta\left(T / T_{c}\right)=$ $\tanh \left\{1.82\left[1.018\left(T_{\mathrm{C}} / T-1\right)\right]^{0.51}\right\}[24,25]$. This gap function is sufficiently precise to explain the temperature dependency at any coupling strength.

The $\lambda^{-2}(T)$ data were fitted based on five different gap models: (a) an isotropic $s$-wave gap, (b) an isotropic $s+s$ wave, (c) anisotropic $s$ wave, (d) a $d$-wave line node, and (e) a nodal $s+d$ wave, as shown in Fig. 1(c). Further, the diamagnetic signal observed below $T_{\mathrm{C}}$ can be seen through the decrease in the internal field below $T_{\mathrm{C}}$ as shown in Fig. 1(d). From the fits presented in Fig. 1(c) it is obvious that the isotropic $s$-wave, anisotropic $s$-wave, and $d$-wave models do not fit the data as they give a high value of goodness of fit $\chi^{2}$ (here $\chi^{2}=\sum\left(\sigma_{\text {obs }}-\sigma_{\text {cal }}\right)^{2} / \sigma_{\text {error }}^{2}(M-N)$, where $M$ is the total data points and $N$ is the number of free parameters). Contrariwise, two-gap models using the isotropic $s+s$ wave and a nodal $s+d$ wave show good fits to the $\lambda^{-2}(T)$ data. Furthermore the low $T$ upturn can be best fitted with a nodal $(s+d)$-wave model with a minimum value of $\chi^{2}=1.3$. The estimated parameters for the nodal $(s+d)$-wave model show one larger gap $2 \Delta_{1}(0) / k_{\mathrm{B}} T_{\mathrm{C}}=6.27 \pm 0.2(\mathrm{meV})$, which is larger than the value of 3.53 as expected for a conventional BCS gap and another smaller gap $2 \Delta_{2}(0) / k_{\mathrm{B}} T_{\mathrm{C}}=1.14 \pm$ $0.1(\mathrm{meV})$. The smaller gap is a nodal gap for the $(s+d)$ wave model. Moreover, the large gap value indicates the presence of strong-coupling superconductivity in $\mathrm{HfV}_{2} \mathrm{Ga}_{4}$. The multigap features are usually seen in iron-based superconductors, $\mathrm{Ba}_{1-x} \mathrm{~K}_{x} \mathrm{Fe}_{2} \mathrm{As}_{2}$ [27,28], cuprate superconductors [29,30], and also in $\mathrm{Bi}_{4} \mathrm{O}_{4} \mathrm{~S}_{3}$ [31]. Following the method described in Ref. [32], we determined the values of $\lambda_{L}(0)=$
797(4) $\mathrm{nm}$ for the $(s+d)$-wave fit, $n_{\mathrm{s}}=6.78(9) \times 10^{25}$ carriers $\mathrm{m}^{-3}$, and $m^{*}=1.528(2) m_{\mathrm{e}}$, respectively, for $\mathrm{HfV}_{2} \mathrm{Ga}_{4}$. The parameters obtained from the fit to the $\sigma_{\mathrm{sc}}(T)$ data of $\mathrm{HfV}_{2} \mathrm{Ga}_{4}$ using different gap models is given in Table I . For detailed calculations, see the Supplemental Material [26].

To examine the fundamental issue of the presence of timereversal symmetry (TRS) breaking or spin fluctuations in $\mathrm{HfV}_{2} \mathrm{Ga}_{4}$, we did ZF- $\mu \mathrm{SR}$ measurements. This technique is extremely helpful to identify the tiny spontaneous magnetic fields below $T_{\mathrm{C}}$. In the case of conventional superconductors, there is no change in the $\mathrm{ZF}$ muon relaxation rate $\left(\lambda_{\mathrm{ZF}}\right)$ below $T_{\mathrm{C}} \cdot \lambda_{\mathrm{ZF}}$ increases with decrease in temperature at $T_{\mathrm{C}}$ if TRS is broken. Figure 2(a) shows the ZF- $\mu$ SR signal at 4.6 and $0.1 \mathrm{~K}$. The ZF- $\mu$ SR signal could be best described by a combined Lorentzian and Gaussian Kubo-Toyabe relaxation function: $A_{\mathrm{ZF}}(t)=A_{2} G_{\mathrm{KT}}(t) e^{-\lambda_{\mathrm{ZF}} t}+A_{\mathrm{bg}}$; here $G_{\mathrm{KT}}(t)=\left[\frac{1}{3}+\frac{2}{3}(1-\right.$ $\left.\left.\sigma_{\mathrm{KT}}^{2} t^{2}\right) \exp \left(-\frac{\sigma_{\mathrm{KT}}^{2} t^{2}}{2}\right)\right]$ is known as the Gaussian Kubo-Toyabe function, and $A_{2}$ and $A_{\mathrm{bg}}$ represent the asymmetry contribution from the sample and the silver holder, respectively. The resulting fit is shown in Fig. 2(b). It is interesting to note that $\lambda_{\mathrm{ZF}}(T)$ increases with decreasing temperature, suggesting the presence of spin fluctuation in $\mathrm{HfV}_{2} \mathrm{Ga}_{4}$. Furthermore, the fits to the ZF data give $\sigma_{\mathrm{KT}}=0.368(5)$ and $\lambda_{\mu}=0.0525(5) \mu \mathrm{s}^{-1}$ at $0.1 \mathrm{~K}$, and $\sigma_{\mathrm{KT}}=0.368(5)$ and $\lambda_{\mu}=0.0337(6) \mu \mathrm{s}^{-1}$ at $4.6 \mathrm{~K}$.

Analysis of the band structure and Fermi surface of the $\mathrm{HfV}_{2} \mathrm{Ga}_{4}$ compound can provide a suitable baseline from which to raise some phenomenological hypothesis of the mechanisms involved in such unconventional superconductivity shown by the experimental evidences presented in this Rapid Communication. Therefore, fulloptimized first-principles calculations were carried out in the framework of the Kohn-Sham scheme within the DFT $[33,34]$, performed using the pseudopotential approach in the Perdew-Burke-Ernzerhof generalized gradient approximation $[35,36]$ as implemented in QUANTUM ESPRESSO [37,38] and supported by auxiliary codes $[39,40]$, to get explicitly the orbital wave-function projections over the Fermi surface.

Figure 3(a) shows the band structure through highsymmetry points in the first Brillouin zone (BZ) [41]. Multiple distinct bands are crossing the Fermi energy $\left(E_{\mathrm{F}}\right)$, with very contrasting $\mathrm{V}-d$ and Hf- $d$ characters. Other atomic orbital contributions to the electronic density in the vicinity of the $E_{\mathrm{F}}$ are negligible. The obtained results are in great agreement with previous electronic-structure calculations [6], which were carried out with a very distinct approach, using full-potential linearized augmented plane waves plus local 


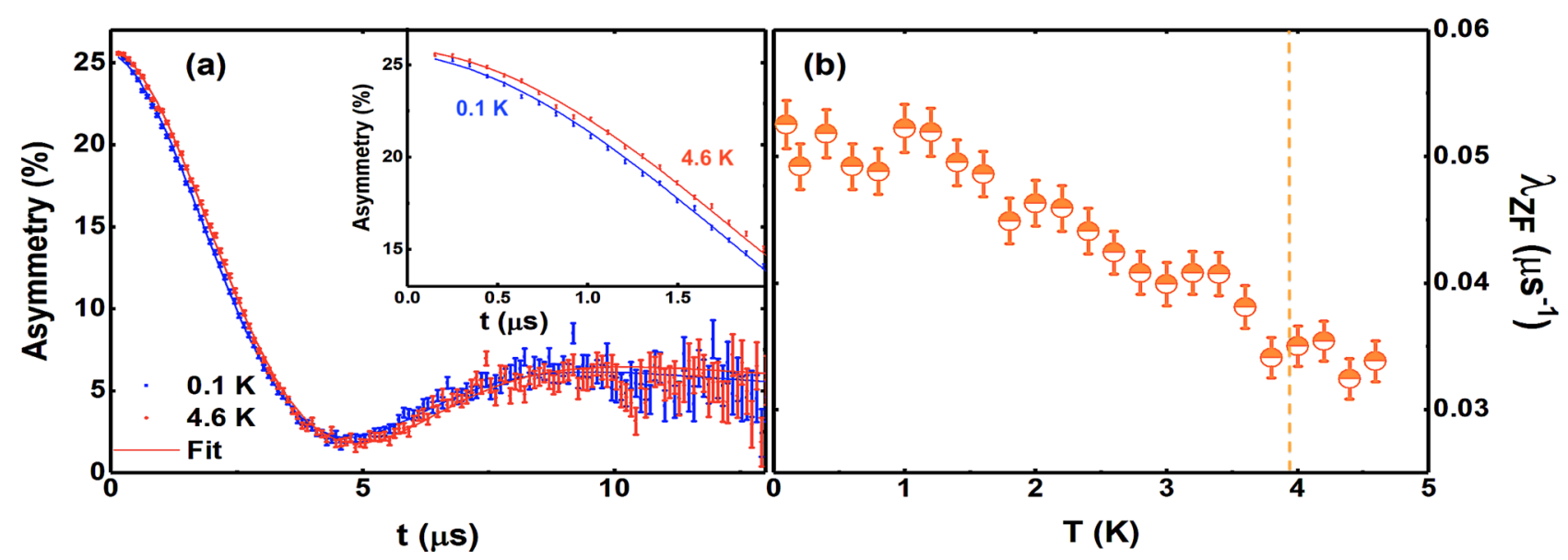

FIG. 2. (a) Represents the ZF- $\mu$ SR spectra for $\mathrm{HfV}_{2} \mathrm{Ga}_{4}$ at 0.1 and $4.6 \mathrm{~K}$. The solid lines are fits to the data, as described in the text. The inset shows the asymmetry data at lower time. (b) shows the temperature dependence of the relaxation rate $\lambda_{\mathrm{ZF}}(T)$, which intimates the presence of spin fluctuations. The vertical dashed line represents $T_{\mathrm{C}}$.

orbitals, except by the presence of an additional band in the Fermi level (resulting in a very small quasispheric hole pocket around the $Z$ point in the Fermi surface). Additionally, Figs. 3(b)-3(d) present the projected-orbital Fermi surface with a color scheme for each irreducible representation. The reddish regions indicate a substantial contribution of $\mathrm{V} d$ orbitals, which dictate the density of states (DOS) at the Fermi level; the bluish ones represent a major character derived from Hf $d$ orbitals, while the greenish branches depict a strong hybridization between these two states, as indicated by the color bar scale.

The Fermi surface consists of multiple tridimensional disconnected sheets, bridging the way for a multiband superconductivity scenario: (a) a minor hybridized hole sphere next to the $Z$ point; (b) a quasihyperbolic paraboloid with hole carriers that develops around the $M$ point with a substantial admixture of Hf- $d$ and V- $d$ orbitals, in the midpoint of the line that connects the two rhombi (which we will call horse saddle), plus a very complex holelike surface around the $Z$ point composed mainly by Hf- $d$ character with a slight degree of V-d (jellyfish); and (c) multiple V-d electron cone-shaped pockets around the $P$ point (seashell), together with small electron pockets saddled within the BZ along the $M-\Gamma$ highsymmetry line.
Such hole bands with a predominant Hf-derived character are deeply sensitive against spin-orbit interactions, as opposed to $\mathrm{V}-d$ bands, which, in turn, play a crucial role in the low-energy electronic states [6]. This fermiology, with very distinct disconnected pockets, in the sense of their orbital weight contribution and, hence, their effective spin-orbit coupling, favors the condensation of pairs with different superconducting order parameters. Since jellyfish pockets have a strong Hf- $d$ character, we could expect the emergence of pair states with nonzero angular momentum and strong angular anisotropy, resulting in an even-parity momentum-dependent order parameter. On the other hand, the negligible effect of SOC in V bands favors the emergence of conventional $s$-wave pairing. This interpretation supports the experimental $(s+d)$ wave symmetry as founded. Moreover, the weighting factor of 0.57 to $s$-wave pairing is attributed to the higher contribution of V states on the DOS at the Fermi level, residing mainly at the seashell pockets, and a lower $d$-wave character coming from those Hf-derived structures.

On the other hand, repulsive interactions between the sheets, driven by spin fluctuations, for instance, are unexpected due to the complex nature of the fermiology. However, the jellyfish and horse-saddle pockets will take advantage of hole doping, increasing the SOC effects on the low-energy
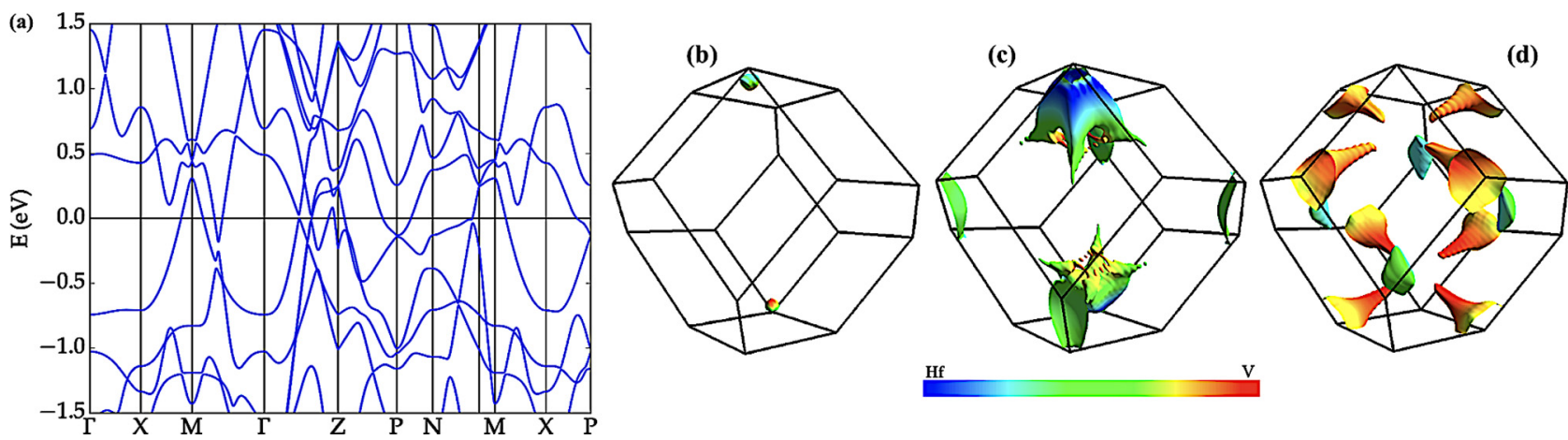

FIG. 3. (a) Electronic bands along a selected path in the first $\mathrm{BZ}$ of $\mathrm{HfV}_{2} \mathrm{Ga}_{4}$. The Fermi energy ( $E_{\mathrm{F}}$ ) is set at $0.0 \mathrm{eV}$. (b)-(d) $\mathrm{Hf}-$ and $\mathrm{V}-d$ orbital character contribution to electronic states projected over the three distinct Fermi surface sheets. 
states, at the same time that seashell pockets will gradually decrease (see the Supplemental Material [26]), paving the way for a discontinuous sign change of the order parameter phase and spin fluctuations by the enlargement of possible nearly induced nesting instabilities [42-45]. This mechanism becomes relevant in our context since Ga-deficient $\mathrm{HfV}_{2} \mathrm{Ga}_{4}$ samples can, in a first approximation, effectively decrease the chemical potential of occupied states by a few meV, promoting such features. Also, hole doping promotes an increase in the density of states, favoring electronic correlations, and contributing to spontaneous symmetry breaking mechanisms. Indeed, nearly nested Fermi surface pockets and the anisotropic gap function support the enhanced pairing strength observed in $\mu \mathrm{SR}$ measurements $\left(2 \Delta_{1} / k_{B} T_{c}=6.27\right)$ [46-48]. Therefore, Ga deficiency can be imperative to the unconventional superconductivity of $\mathrm{HfV}_{2} \mathrm{Ga}_{4}$, which seems to be unique, and high-quality single crystals, with systematic doping content, are urgently required to investigate the superconducting state further and to confirm the proposed hypothesis.

In summary, we have examined the superconducting state of $\mathrm{HfV}_{2} \mathrm{Ga}_{4}$ utilizing magnetotransport, $\mu \mathrm{SR}$, and numerical band-structure calculations. The temperature dependence of the magnetic penetration depth, $\lambda^{-2}(T)$, is better fitted to a nodal two-gap $(s+d)$-wave model than a single-gap isotropic $s$-wave, anisotropic $s$-wave, or $d$-wave model, suggesting unconventional superconductivity in $\mathrm{HfV}_{2} \mathrm{Ga}_{4}$. The large value of gap to $T_{\mathrm{C}}$ ratio, $2 \Delta_{1}(0) / k_{\mathrm{B}} T_{\mathrm{C}}=6.27 \pm 0.2$, obtained from the nodal $(s+d)$-wave gap, is larger than 3.53, expected for conventional BCS superconductors, indicating the presence of strong-coupling superconductivity in $\mathrm{HfV}_{2} \mathrm{Ga}_{4}$. The decrease in the $\mathrm{ZF}$ relaxation rate with increasing temperature indicates the presence of spin fluctuations in the superconducting state of $\mathrm{HfV}_{2} \mathrm{Ga}_{4}$. In addition, $a b$ initio calculations suggest that there are electrons derived from multiple distinct bands in disconnected sheets of the Fermi surface, in agreement with the experimental evidence of two-gap superconductivity for $\mathrm{HfV}_{2} \mathrm{Ga}_{4}$, and the momentum-dependent SOC interactions may be a theoretical starting point to elucidate the appearance of nodal superconductivity.

The authors acknowledge Kartik Panda for helping with MUSR data analysis and Professor A. M. Strydom for interesting discussions. A.B. would like to acknowledge the Department of Science and Technology (DST), India, for an Inspire Faculty Research Grant (Grant No. DST/INSPIRE/04/2015/000169), and the UK-India Newton grant for funding support. D.T.A. would like to thank the Royal Society of London for the UK-China Newton funding and the Japan Society for the Promotion of Science for support from an invitation fellowship. F.B.S., P.P.F., L.E.C., A.J.S.M., A.L.R.M., and L.T.F.E. gratefully acknowledge the financial support of the Brazilian agencies Conselho Nacional de Desenvolvimento Cientifico e Tecnologico (CNPq) under Grants No. 302149/2017-1 and No. 431868/2018-2, and Fundacão de Amparo à Pesquisa do Estado de São Paulo (FAPESP) under Grants No. 2016/11774-5, No. 2016/11565-7, No. 2016/10167-8, No. 2018/08819-2, No. 2018/10835-6, No. 2018/20546-1, No. 2019/05005-7, and No. 2019/07082-9.
[1] D. J. Scalapino, Rev. Mod. Phys. 84, 1383 (2012).

[2] G. Stewart, Adv. Phys. 66, 75 (2017).

[3] M. Norman, Novel Superfluids, edited by K. H. Bennemann and J. B. Ketterson (Oxford University Press, Oxford, 2014), Vol. 2, pp. 23-79.

[4] F. B. Santos, L. E. Correa, B. S. de Lima, O. V. Cigarroa, M. S. da Luz, T. W. Grant, Z. Fisk, and A. J. S. Machado, Phys. Lett. A 382, 1065 (2018).

[5] N. R. Werthamer, E. Helfand, and P. C. Hohenberg, Phys. Rev. 147, 295 (1966).

[6] P. P. Ferreira, F. B. Santos, A. J. S. Machado, H. M. Petrilli, and L. T. F. Eleno, Phys. Rev. B 98, 045126 (2018).

[7] P. Ferreira, T. Dorini, F. Santos, A. Machado, and L. Eleno, Materialia 4, 529 (2018).

[8] A. Sauer, D. A. Zocco, A. H. Said, R. Heid, A. Böhmer, and F. Weber, Phys. Rev. B 99, 134511 (2019).

[9] B. Wang and K. Ohgushi, Sci. Rep. 3, 3381 (2013).

[10] J. E. Sonier, J. H. Brewer, and R. F. Kiefl, Rev. Mod. Phys. 72, 769 (2000).

[11] F. L. Pratt, Physica B 289-290, 710 (2000).

[12] A. J. S. Machado, N. P. Baptista, B. S. de Lima, N. Chaia, T. W. Grant, L. E. Corrêa, S. T. Renosto, A. C. Scaramussa, R. F. Jardim, M. S. Torikachvili, J. A. Aguiar, O. C. Cigarroa, L. T. F. Eleno, and Z. Fisk, Phys. Rev. B 95, 144505 (2017).

[13] E. M. Chudnovsky, Phys. Rev. B 95, 100503(R) (2017).

[14] M. Abdel-Hafiez, J. Ge, A. N. Vasiliev, D. A. Chareev, J. Van de Vondel, V. V. Moshchalkov, and A. V. Silhanek, Phys. Rev. B 88, 174512 (2013).
[15] C. Ren, Z. S. Wang, H. Q. Luo, H. Yang, L. Shan, and H. H. Wen, Phys. C (Amsterdam, Neth. 469, 599 (2009).

[16] C. Ren, Z. S. Wang, H. Q. Luo, H. Yang, L. Shan, and H. H. Wen, Phys. Rev. Lett. 101, 257006 (2008).

[17] S. L. Li, H. H. Wen, Z. W. Zhao, Y. M. Ni, Z. A. Ren, G. C. Che, H. P. Yang, Z. Y. Liu, and Z. X. Zhao, Phys. Rev. B 64, 094522 (2001).

[18] A. Bhattacharyya, D. T. Adroja, M. Smidman, and V. K. Anand, Sci. China: Phys., Mech. Astron. 61, 127402 (2018).

[19] D. Adroja, A. Bhattacharyya, P. K. Biswas, M. Smidman, A. D. Hillier, H. Mao, H. Luo, G. H. Cao, Z. Wang, and C. Wang, Phys. Rev. B 96, 144502 (2017).

[20] A. Bhattacharyya, D. T. Adroja, K. Panda, S. Saha, T. Das, A. J. S. Machado, T. W. Grant, Z. Fisk, A. D. Hillier, and P. Manfrinetti, Phys. Rev. Lett. 122, 147001 (2019).

[21] A. Bhattacharyya, D. T. Adroja, J. Quintanilla, A. D. Hillier, N. Kase, A. M. Strydom, and J. Akimitsu, Phys. Rev. B 91, 060503(R) (2015).

[22] R. Prozorov and R. W. Giannetta, Supercond. Sci. Technol. 19, R41 (2006).

[23] D. T. Adroja, A. Bhattacharyya, M. Telling, Y. Feng, M. Smidman, B. Pan, J. Zhao, A. D. Hillier, F. L. Pratt, and A. M. Strydom, Phys. Rev. B 92, 134505 (2015).

[24] G. M. Pang, M. Smidman, W. B. Jiang, J. K. Bao, Z. F. Weng, Y. F. Wang, L. Jiao, J. L. Zhang, G. H. Cao, and H. Q. Yuan, Phys. Rev. B 91, 220502(R) (2015).

[25] J. F. Annett, Adv. Phys. 39, 83 (1990). 
[26] See Supplemental Material at http://link.aps.org/supplemental/ 10.1103/PhysRevResearch.2.022001 for more information on the crystal structure, resistivity, and magnetization, and the Uemura plot.

[27] D. V. Evtushinsky, D. S. Inosov, V. B. Zabolotnyy, M. S. Viazovska, R. Khasanov, A. Amato, H.-H. Klauss, H. Luetkens, Ch Niedermayer, G. L. Sun, V. Hinkov, C. T. Lin, A. Varykhalov, A. Koitzsch, M. Knupfer, B. Chner, A. A. Kordyuk, and S. V. Borisenko, New J. Phys. 11, 055069 (2009).

[28] R. Khasanov, D. V. Evtushinsky, A. Amato, H.-H. Klauss, H. Luetkens, Ch. Niedermayer, B. Buchner, G. L. Sun, C. T. Lin, J. T. Park, D. S. Inosov, and V. Hinkov, Phys. Rev. Lett. 102, 187005 (2009).

[29] R. Khasanov, S. Strassle, D. Di Castro, T. Masui, S. Miyasaka, S. Tajima, A. Bussmann-Holder, and H. Keller, Phys. Rev. Lett. 99, 237601 (2007).

[30] B. Keimer, S. A. Kivelson, M. R. Norman, S. Uchida, and J. Zaanen, Nature (London) 518, 179 (2015).

[31] P. K. Biswas, A. Amato, C. Baines, R. Khasanov, H. Luetkens, H. Lei, C. Petrovic, and E. Morenzon, Phys. Rev. B 88, 224515 (2013).

[32] A. Hillier and R. Cywinski, Appl. Magn. Reson. 13, 95 (1997).

[33] P. Hohenberg and W. Kohn, Phys. Rev. 136, B864 (1964).

[34] W. Kohn and L. J. Sham, Phys. Rev. 140, A1133 (1965).
[35] J. P. Perdew, K. Burke, and M. Ernzerhof, Phys. Rev. Lett. 77, 3865 (1996).

[36] A. Dal Corso, Comput. Mater. Sci. 95, 337 (2014).

[37] P. Giannozzi, S. Baroni, N. Bonini, M. Calandra, R. Car, C. Cavazzoni, D. Ceresoli, G. L. Chiarotti, M. Cococcioni, I. Dabo et al., J. Phys.: Condens. Matter 21, 395502 (2009).

[38] P. Giannozzi, O. Andreussi, T. Brumme, O. Bunau, M. B. Nardelli, M. Calandra, R. Car, C. Cavazzoni, D. Ceresoli, M. Cococcioni et al., J. Phys.: Condens. Matter 29, 465901 (2017).

[39] M. Kawamura, Comput. Phys. Commun. 239, 197 (2019).

[40] A. Kokalj, J. Mol. Graphics Modell. 17, 176 (1999).

[41] W. Setyawan and S. Curtarolo, Comput. Mater. Sci. 49, 299 (2010).

[42] K. Kuroki and R. Arita, Phys. Rev. B 64, 024501 (2001).

[43] I. I. Mazin, D. J. Singh, M. D. Johannes, and M. H. Du, Phys. Rev. Lett. 101, 057003 (2008).

[44] K. Kuroki, S. Onari, R. Arita, H. Usui, Y. Tanaka, H. Kontani, and H. Aoki, Phys. Rev. Lett. 101, 087004 (2008).

[45] D. J. Singh, New J. Phys. 14, 123003 (2012).

[46] K. Terashima, Y. Sekiba, J. Bowen, K. Nakayama, T. Kawahara, T. Sato, P. Richard, Y.-M. Xu, L. Li, G. Cao et al., Proc. Natl. Acad. Sci. USA 106, 7330 (2009).

[47] P. Monthoux and D. J. Scalapino, Phys. Rev. B 50, 10339 (1994).

[48] C.-H. Pao and N. E. Bickers, Phys. Rev. Lett. 72, 1870 (1994). 